\title{
BMJ Open Quantifying sociodemographic and income disparities in medical therapy and lifestyle among symptomatic patients with suspected coronary artery disease: a cross-sectional study in North America
}

\author{
Joseph A Ladapo, ${ }^{1}$ Adrian Coles, ${ }^{2}$ Rowena J Dolor, ${ }^{2}$ Daniel B Mark, ${ }^{2}$ \\ Lawton Cooper, ${ }^{3}$ Kerry L Lee, ${ }^{2}$ Jonathan Goldberg, ${ }^{4}$ Michael D Shapiro, ${ }^{5}$ \\ Udo Hoffmann, ${ }^{6}$ Pamela S Douglas ${ }^{2}$
}

To cite: Ladapo JA, Coles A, Dolor RJ, et al. Quantifying sociodemographic and income disparities in medical therapy and lifestyle among symptomatic patients with suspected coronary artery disease: a cross-sectional study in North America. BMJ Open 2017;7:e016364. doi:10.1136/ bmjopen-2017-016364

- Prepublication history and additional material for this paper are available online. To view, please visit the journal (http:// dx.doi.org/bmjopen-2017016364).

Received 10 February 2017 Revised 19 July 2017 Accepted 25 July 2017

CrossMark

For numbered affiliations see end of article.

Correspondence to

Dr Joseph A Ladapo; jladapo@mednet.ucla.edu

\section{ABSTRACT}

Objectives To evaluate potential gaps in preventive medical therapy and healthy lifestyle practices among symptomatic patients with suspected coronary artery disease (CAD) seeing primary care physicians and cardiologists and how gaps vary by sociodemographic characteristics and baseline cardiovascular risk. Design Cross-sectional study assessing potential preventive gaps.

Participants 10003 symptomatic outpatients evaluated by primary care physicians, cardiologists or other specialists for suspected CAD.

Setting PROspective Multicenter Imaging Study for Evaluation of Chest Painfrom 2010 to 2014.

Measures Primary measures were absence of an antihypertensive, statin or angiotensin-converting enzyme inhibitor/angiotensin receptor blocker for renal protection in patients with hypertension, dyslipidaemia or diabetes, respectively, and being sedentary, smoking or being obese. Results Preventive treatment gaps affected 14\% of patients with hypertension, $36 \%$ of patients with dyslipidaemia and $32 \%$ of patients with diabetes. Overall, $49 \%$ of patients were sedentary, $18 \%$ currently smoked and $48 \%$ were obese. Women were significantly more likely to not take a statin for dyslipidaemia and to be sedentary. Patients with lower socioeconomic status were also significantly more likely to not take a statin. Compared with Whites, Blacks were significantly more likely to be obese, while Asians were less likely to smoke or be obese. High-risk patients sometimes experienced larger preventive care gaps than low-risk patients. For patients with dyslipidaemia, the presence of a treatment gap was associated with a higher risk of an adverse event (HR 1.35, $95 \% \mathrm{Cl} 1.02$ to 1.82 ).

Conclusions Among contemporary, symptomatic patients with suspected CAD, significant gaps exist in preventive care and lifestyle practices, and high-risk patients sometimes had larger gaps. Differences by sex, age, race/ethnicity, socioeconomic status and geography are modest but contribute to disparities and have
Strengths and limitations of this study

- The study had a large sample size with an $\mathrm{N}$ of 10003 patients.

- Measures of preventive lifestyle practices were gathered through self-report; errors or inaccuracies in self-report could therefore affect our results.

- There may have been patients whose hypertension or dyslipidaemia were well controlled with dietary changes and exercise alone.

- Among patients with diabetes, we did not have clinical information about albuminuria, so there may have been patients for whom the benefit of angiotensin-converting enzyme inhibitor or angiotensin receptor blocker therapy was uncertain.

- Use of body mass index (BMI) as a surrogate for body fatness and obesity identification is also vulnerable to misclassification, since sex, age, race/ ethnicity and muscle mass influence the relationship between $\mathrm{BMI}$ and excess fat.

implications for improving opulation health. For patients with dyslipidaemia, the presence of a treatment gap was associated with a higher risk of an adverse event. Clinical trial registration Clinical Trials.gov identifier NCT01174550.

Preventive medical care and lifestyle practices reduce the risk of adverse cardiovascular events ${ }^{12}$ and may influence how likely a patient is to present to their primary care physician or cardiologist with symptoms suggestive of coronary artery disease (CAD). In the USA, approximately 4 million of these patients are referred for outpatient cardiac stress testing or coronary CT angiography (CTA) each year. ${ }^{3}$ Although most have significant risk factors 
for adverse cardiovascular events, such as hypertension, dyslipidaemia and diabetes, ${ }^{45}$ little is known about their preventive medical and lifestyle practices prior to presentation, the extent to which these preventive measures differ from national recommendations and guidelines ${ }^{6-9}$ or their relationship with sociodemographic and socioeconomic disparities. Understanding these patterns and characterising the magnitude of medical or lifestyle gaps-that is, the difference between recommended preventive care and actual preventive care-is a critical step toward preventing disease and reducing adverse cardiovascular events in this population, independent of the outcome of diagnostic testing. Further, if preventive care varies by sociodemographic characteristics, this variation may contribute to important health disparities and identify populations in need of specific targeting. To identify opportunities for improving preventive care in this population, we used data from symptomatic patients in the PROspective Multicenter Imaging Study for Evaluation of Chest Pain (PROMISE) to (1) evaluate potential gaps in preventive medical therapy among patients with hypertension, dyslipidaemia or diabetes; (2) determine the extent to which these gaps differed by patients' baseline risk; (3) evaluate gaps in healthy lifestyle practices, as defined by being sedentary, smoking or being obese and (4) determine which gaps vary by sex, age, race/ethnicity, socioeconomic status and geography.

\section{METHODS}

\section{Study design}

Methods used in PROMISE have been described previously. ${ }^{410}$ The study protocol was approved by the local or central institutional review board at each coordinating centre and at each enrolling site in North America. We enrolled symptomatic outpatients without diagnosed CAD whose physicians believed that non-urgent, non-invasive cardiovascular testing was necessary for the evaluation of suspected CAD. After providing written informed consent, 10003 eligible patients were randomly assigned to either anatomical testing with CTA or functional testing with exercise electrocardiography, nuclear stress or stress echocardiography. ${ }^{10}$ Enrollment began on 27 July 2010 and was completed on 19 September 2013. All the patients were followed until 31 October 2014. Analyses were performed in 2016.

\section{Gaps in preventive medications and lifestyle practices}

At the time of enrollment, information about preventive medication use and lifestyle practices was collected by the clinical sites through patient report, chart review and other clinical sources. We focused on six potential gaps in preventive care that have been demonstrated to increase the risk of cardiovascular disease ${ }^{111}$ : absence of an antihypertensive medication in patients with hypertension, absence of a statin in patients with dyslipidaemia, absence of an angiotensin-converting enzyme inhibitor or angiotensin receptor blocker (ACEi/ARB) for renal protection in patients with diabetes, being sedentary, smoking and being obese, as determined by a body mass index (BMI) exceeding 30. Because patients had to be eligible for randomisation to either CTA or functional testing, no patients known to have renal dysfunction were enrolled.

\section{Hypertension, dyslipidaemia and diabetes}

Because PROMISE was a pragmatic trial, diagnoses were identified and defined by physicians at the participating clinics rather than with study-specific criteria. Among our symptomatic patients, absence of antihypertensive medication in patients with hypertension was defined as a preventive care gap because of evidence that treating patients with this comorbidity reduces the risk of cardiovascular events ${ }^{12}$ and because treatment is consistent with recommendations issued by the American Society of Hypertension, International Society of Hypertension $^{13}$ and American Heart Association. ${ }^{14}$ Absence of a statin in patients with dyslipidaemia was considered a preventive care gap because statin use in primary and secondary prevention has been shown to reduce cardiovascular risk. ${ }^{1516}$ The median atherosclerotic cardiovascular disease (ASCVD) score in our population was 11.3\% with an IQR of $6.1 \%$ to $19.8 \%$, well above the 10 -year risk threshold of $7.5 \%$ for treatment in most participants. ${ }^{17}$ Applying lower ASCVD thresholds for statin therapy has also been shown to be cost-effective. ${ }^{18}$ Absence of an ACEi/ARB for renal protection in patients with diabetes was considered a preventive care gap because the vast majority of diabetics in our population were hypertensive $(79.9 \%)$ and prophylactic use of ACEi/ARBs reduces the incidence of albuminuria, ${ }^{19-21}$ which has been shown to be a risk factor for cardiovascular ${ }^{22}$ and overall mortality in patients with diabetes. ${ }^{23} 24$

\section{Physical inactivity, smoking and obesity}

Being sedentary, smoking and being obese have all been demonstrated to increase cardiovascular risk and therefore represent important gaps in preventive lifestyle practices. ${ }^{1}$ We assessed the prevalence of these lifestyle practices across all patients in our cohort. To assess activity level, we asked, "During the past month, did you participate in any physical activities or exercise regularly (one or more times per week)? Examples include: running, aerobics, golf, gardening, walking, etc" (yes or no). To assess smoking, we asked, "Have you smoked in the past 2 weeks?" (yes or no).

\section{Demographics and socioeconomics}

We focused on disparities in prevention by sex, age, race/ ethnicity, socioeconomic status and geography. Race/ ethnicity was reported by the patient and categorised into the following mutually exclusive groups: White; Black; American Indian/Alaska Native, Native Hawaiian/Other Pacific Islanders; Asian (not including any Hispanics) and Hispanics (from any racial/ethnic group) ${ }^{25}$ Socioeconomic status was defined by the median household income of the patient's zip code based on data extracted 
from 2010 US Census Bureau American Community Survey Data (5-year estimates), similar to prior work. ${ }^{26-29}$ Socioeconomic status was categorised into quartiles from lowest to highest median household income (low, medium low, medium high and high). We used the following US Census categories for geographic regions: Northeast, Midwest, West and South.

\section{Statistical analysis}

Analyses were based on patient status at presentation for CAD evaluation. $p$ Values of less than 0.05 were considered significant. We estimated summary statistics for gaps in preventive care and lifestyle practices and constructed multivariable logistic regression models to assess the association of patients' sociodemographic characteristics (sex, age, race/ethnicity, socioeconomic status and geography) at presentation with these gaps, while controlling for baseline risk (for blood pressure: systolic $<140 \mathrm{~mm}$ $\mathrm{Hg}$ and diastolic $<90 \mathrm{~mm} \mathrm{Hg}$, systolic 140 to $159 \mathrm{~mm} \mathrm{Hg}$ or diastolic 90 to $99 \mathrm{~mm} \mathrm{Hg}$, and systolic $\geq 160 \mathrm{~mm} \mathrm{Hg}$ or diastolic $\geq 100 \mathrm{~mm} \mathrm{Hg}$; for ASCVD: $<7.5 \%, 7.5 \%$ to $<15 \%$ and $\geq 15 \%$ ), other clinical characteristics and physician specialty (see online supplementary appendix tables 1.1-1.3 for detailed clinical characteristics and online supplementary appendix tables 2.1 and 2.2 for fully reported regression results). In addition to estimating covariate-adjusted ORs and their corresponding 95\% CIs, the fitted models were used to compute covariate-adjusted probabilities ${ }^{30}$ (also known as 'predictive margins') of gaps in preventive medication use and healthy lifestyle practices, with stratification by sociodemographic characteristics. In these analyses, the regression models predict proportions for each sociodemographic characteristic, while holding the distribution of all other covariates constant. We excluded $4 \%$ of patients in PROMISE from our multivariable analyses because we were unable to match their reported zip codes to US Census Bureau data. Statistical analyses were performed using SAS V.9.2 or higher (SAS Institute).

\section{RESULTS}

\section{Symptomatic patients: characteristics and baseline risk}

Characteristics of the 10003 symptomatic patients $(88 \%$ with chest pain/dyspnoea, $12 \%$ with other symptoms) presenting to their primary care physicians, cardiologists or other specialists are summarised in table 1 . The median age of the cohort was 60.0 years (IQR, 54.4-66.0 years), and $52.7 \%$ were women. Whites composed $77.4 \%$ of the cohort, and Blacks and Hispanics composed $10.8 \%$ and $7.7 \%$, respectively. Asians composed $2.5 \%$ of the cohort, and people of other/unknown race/ethnicity composed $1.6 \%$ of the cohort. Patients in the lowest socioeconomic quartile lived in zip codes with a median household income less than US $\$ 42610$, while patients in the highest socioeconomic quartile lived in zip codes with median household income of at least US\$71059 annually.
Table 1 Demographics and presenting characteristics for all patients

\begin{tabular}{|c|c|}
\hline Characteristic & $\begin{array}{l}\text { All patients } \\
(n=10003)\end{array}$ \\
\hline Female (\%) & $5270 / 10003(52.7 \%)$ \\
\hline Median age (IQR), years & $60.0(54.4-66.0)$ \\
\hline \multicolumn{2}{|l|}{ Age (\%), years } \\
\hline $45-64$ & $7111 / 10003$ (71.1\%) \\
\hline $65-79$ & $2711 / 10003(27.1 \%)$ \\
\hline $80+$ & $181 / 10003$ (1.8\%) \\
\hline \multicolumn{2}{|l|}{ Race/ethnicity (\%) } \\
\hline Hispanic or Latino & $767 / 9945(7.7 \%)$ \\
\hline Not Hispanic or Latino-White & $7693 / 9945(77.4 \%)$ \\
\hline Not Hispanic or Latino-Black & $1071 / 9945(10.8 \%)$ \\
\hline Not Hispanic or Latino-Asian & $250 / 9945(2.5 \%)$ \\
\hline Not Hispanic or Latino-Other & $164 / 9945(1.6 \%)$ \\
\hline \multicolumn{2}{|l|}{$\begin{array}{l}\text { Socioeconomic status (minimum, } \\
\text { maximum income), } \$^{*}\end{array}$} \\
\hline Low & 11118,42610 \\
\hline Medium-low & 42613,54149 \\
\hline Medium-high & 54167,71034 \\
\hline High & 71059,184338 \\
\hline \multicolumn{2}{|l|}{ US region† } \\
\hline Midwest & $2208 / 9690(22.8 \%)$ \\
\hline Northeast & $1439 / 9690(14.9 \%)$ \\
\hline South & $3999 / 9690(41.3 \%)$ \\
\hline West & $2044 / 9690(21.1 \%)$ \\
\hline \multicolumn{2}{|l|}{ Cardiac risk factors } \\
\hline $\begin{array}{l}\mathrm{BMI} \geq 30(\%) \text { (median 29.7, IQR } \\
26.3-33.9)\end{array}$ & $4724 / 9907(47.7 \%)$ \\
\hline Hypertension (\%) & $6501 / 10002(65.0 \%)$ \\
\hline Diabetes (\%) & $2144 / 10002(21.4 \%)$ \\
\hline Dyslipidaemia (\%) & $6767 / 10002(67.7 \%)$ \\
\hline $\begin{array}{l}\text { Family history of premature CAD } \\
(\%)\end{array}$ & $3202 / 9970(32.1 \%)$ \\
\hline $\begin{array}{l}\text { Peripheral arterial disease or } \\
\text { cerebrovascular disease (\%) }\end{array}$ & $552 / 10003(5.5 \%)$ \\
\hline CAD risk equivalent (\%) & $2531 / 10003(25.3 \%)$ \\
\hline Metabolic syndrome (\%) & $3772 / 10003(37.7 \%)$ \\
\hline Current tobacco use (\%) & $1773 / 10000(17.7 \%)$ \\
\hline Regular exercise (\%) & $5116 / 9982(51.3 \%)$ \\
\hline History of depression (\%) & 2058/10 000 (20.6\%) \\
\hline \multicolumn{2}{|l|}{ Risk scores } \\
\hline $\begin{array}{l}\text { Mean Diamond and Forrester } \\
\text { score (SD) [n] }\end{array}$ & 53 (20.14) [10003] \\
\hline $\begin{array}{l}\text { Mean ASCVD Pooled Cohort Risk } \\
\text { Prediction (2013) (SD) [n] }\end{array}$ & 15 (11.75) [9901] \\
\hline \multicolumn{2}{|l|}{ Medication use (\%) } \\
\hline Aspirin & $4280 / 9569$ (44.7\%) \\
\hline
\end{tabular}


Table 1 Continued

\begin{tabular}{ll}
\hline Characteristic & $\begin{array}{l}\text { All patients } \\
\text { (n=10003) }\end{array}$ \\
\hline Statin & $4389 / 9569(45.9 \%)$ \\
Beta-blocker & $2399 / 9569(25.1 \%)$ \\
\hline ACE inhibitor or ARB & $4194 / 9569(43.8 \%)$ \\
\hline Primary presenting symptoms (\%) & \\
\hline Chest pain & $7272 / 9996(72.7 \%)$ \\
\hline Dyspnoea & $1490 / 9996(14.9 \%)$ \\
\hline Other & $1234 / 9996(12.3 \%)$ \\
\hline Type of angina (\%) & \\
\hline Typical & $1166 / 10003(11.7 \%)$ \\
\hline Atypical & $7773 / 10003(77.7 \%)$ \\
\hline Non-cardiac & $1064 / 10003(10.6 \%)$ \\
\hline Physician specialty (\%) & \\
\hline Cardiology & $8662 / 10003(86.6 \%)$ \\
\hline Internal medicine & $565 / 10003(5.6 \%)$ \\
\hline Other & $776 / 10003(7.8 \%)$ \\
\hline
\end{tabular}

*Median household income (in US\$) is used as a surrogate for socioeconomic status.

†143 patients had missing zip code data, and 170 patients had zip codes that were not reported in 2010 Census American Community Survey data.

$\mathrm{ACE}$, angiotensin-converting enzyme; ARB, angiotensin receptor blocker; ASCVD, atherosclerotic cardiovascular disease; BMI, body mass index; CAD, coronary artery disease.

\section{Preventive medical and lifestyle gaps}

Overall, the prevalences of hypertension, dyslipidaemia and diabetes were $65.0 \% \quad(n=6501), 67.7 \% \quad(n=6767)$ and $21.4 \% \quad(\mathrm{n}=2144)$, respectively. Among these symptomatic patients, preventive treatment gaps affected $14 \%$ of patients with hypertension, $36 \%$ of patients with dyslipidaemia and $32 \%$ of patients with diabetes. In our examination of preventive lifestyle practices, $49 \%$ of all patients were sedentary, $18 \%$ were current smokers and $48 \%$ were obese.

\section{Association of preventive care gaps with sex, age and race/ ethnicity}

Adjusted ORs for the association between patient characteristics and preventive care gaps are presented in table 2 and covariate-adjusted probabilities of preventive care gaps are presented in figures 1 and 2 . Women were significantly more likely than men to not take a statin for dyslipidaemia (OR 1.33, 95\% CI 1.18 to 1.50 ) and to be sedentary (OR $1.55,95 \%$ CI 1.41 to 1.70 ). Older patients were significantly less likely than the youngest patients to not be taking a statin for dyslipidaemia (65-79 years: OR $0.64,95 \%$ CI 0.55 to 0.75 ; $\geq 80$ years: OR 0.59 , $95 \% \mathrm{CI} 0.38$ to 0.92$)$ and to smoke ( $65-79$ years: OR 0.23 , $95 \%$ CI 0.19 to $0.27 ; \geq 80$ years: OR $0.04,95 \%$ CI 0.01 to $0.13)$. There were no significant differences in preventive medications by patients' race/ethnicity, but differences existed in preventive lifestyle practices: compared with
White patients, Blacks were significantly more likely to be obese (OR 1.55, 95\% CI 1.31 to 1.84), while Asians were less likely to smoke (OR $0.49,95 \%$ CI 0.30 to 0.80 ) or be obese (OR $0.16,95 \%$ CI 0.11 to 0.24 ). There were no significant differences in preventive lifestyle practices of Hispanics compared with Whites.

\section{Variation in preventive care gaps between higher and lower risk symptomatic patients}

The prevalence of preventive medical therapy gaps varied by patient risk. Among symptomatic patients with hypertension, those at the highest overall cardiovascular risk (ASCVD $\geq 15 \%$ ) were less likely to not be on an antihypertensive than patients at the lowest overall cardiovascular risk (ASCVD $<7.5 \%$ ) (OR $0.45,95 \%$ CI 0.34 to 0.58$)$, but patients with the highest blood pressure $(\geq 160 / 100)$ were more likely to not be on an antihypertensive than patients with the lowest blood pressure $(<140 / 90)$ (OR $1.54,95 \%$ CI 1.19 to 1.99 ). Among patients with dyslipidaemia, those at the highest overall cardiovascular risk (OR 1.22, 95\% CI 1.01 to 1.47 ) and with the highest blood pressure (OR 1.28, 95\% CI 1.03 to 1.59) were more likely to not be on a statin, compared with patients with the lowest cardiovascular risk or lowest blood pressure. Among patients with diabetes, those at the highest overall cardiovascular risk were less likely to not be on an ACEi/ $\mathrm{ARB}$ than patients at the lowest overall cardiovascular risk (OR $0.64,95 \%$ CI 0.42 to 0.97 ) (see online supplementary appendix table 2.1).

For the combined endpoint of death, myocardial infarction or hospitalisation for unstable angina, there was no association between having a treatment gap and the risk of an adverse event among patients with hypertension or diabetes. However, for patients with dyslipidaemia, the presence of a treatment gap was associated with a higher risk of an adverse event (HR 1.35, 95\% CI 1.02 to 1.82 ).

\section{Association of preventive care gaps with socioeconomic status/geography}

Compared with symptomatic patients with the highest socioeconomic status, patients with a medium-high socioeconomic status were more likely to not receive an antihypertensive for hypertension (OR 1.25, 95\% CI 1.01 to 1.55 ), while patients with the lowest socioeconomic status were more likely to not receive a statin for dyslipidaemia (OR 1.20, 95\% CI 1.02 to 1.41) (table 2, figure 2). Patients with lower socioeconomic status were also more likely to be sedentary (low: OR 1.45, 95\% CI 1.28 to 1.65; medium low: OR 1.20, 95\% CI 1.07 to 1.36 ; medium high: OR $1.18,95 \%$ CI 1.05 to 1.32 ) and smoke (low: OR 2.00, 95\% CI 1.68 to 2.38 ; medium low: OR $1.63,95 \%$ CI 1.38 to 1.94 ; medium high: OR $1.52,95 \%$ CI 1.28 to 1.80 ) than patients with the highest socioeconomic status (table 2), and these differences were more pronounced as socioeconomic status fell. Regional differences were common: compared with patients in the South, patients living in the West were more likely to not receive antihypertensives for hypertension (OR 1.32, 95\% CI 1.08 to 1.63) and not 


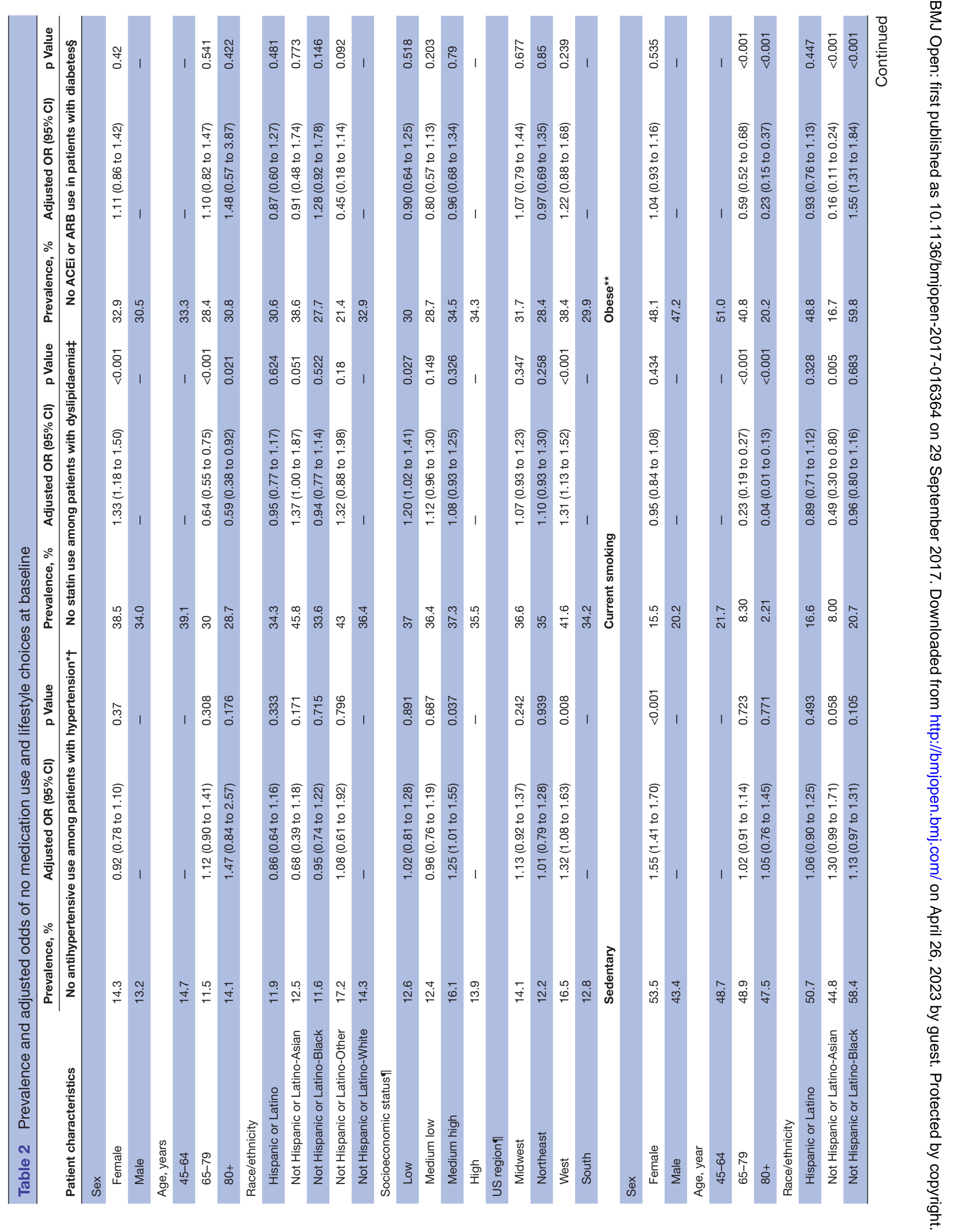




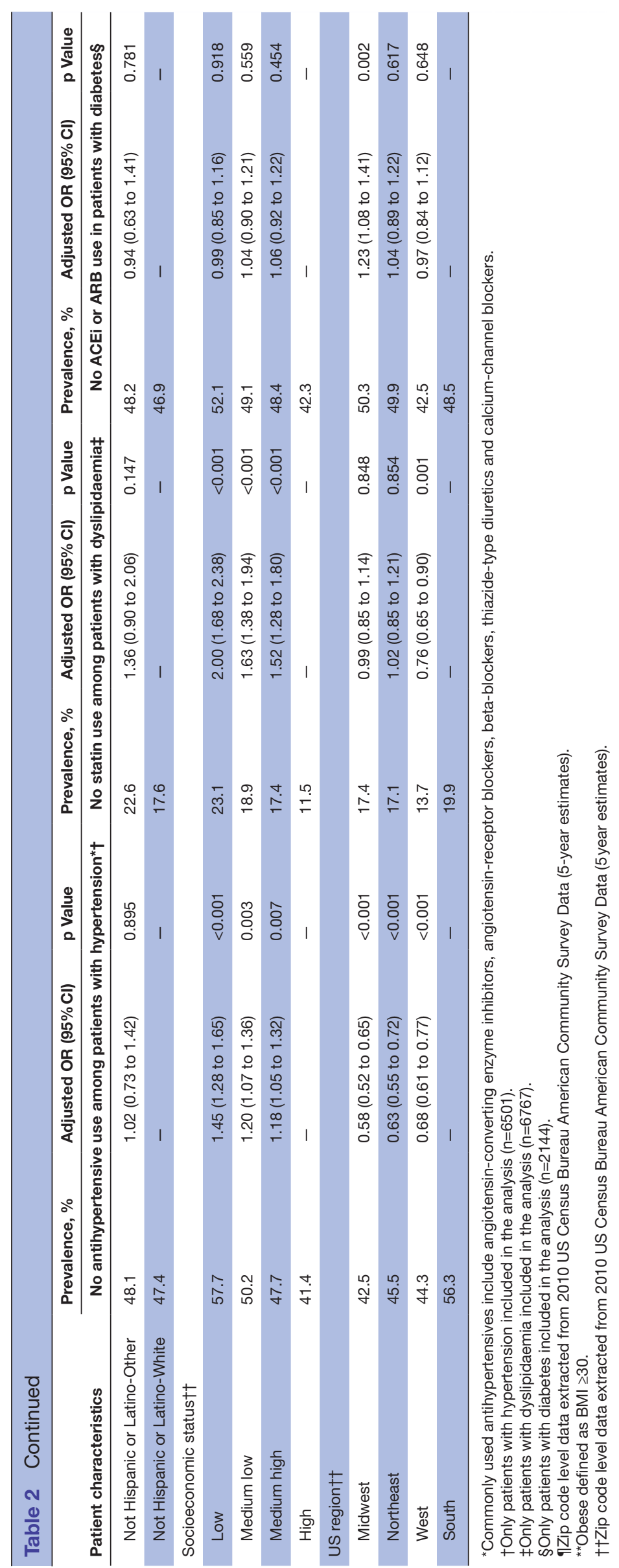

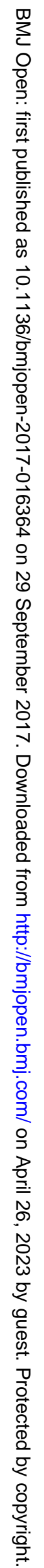



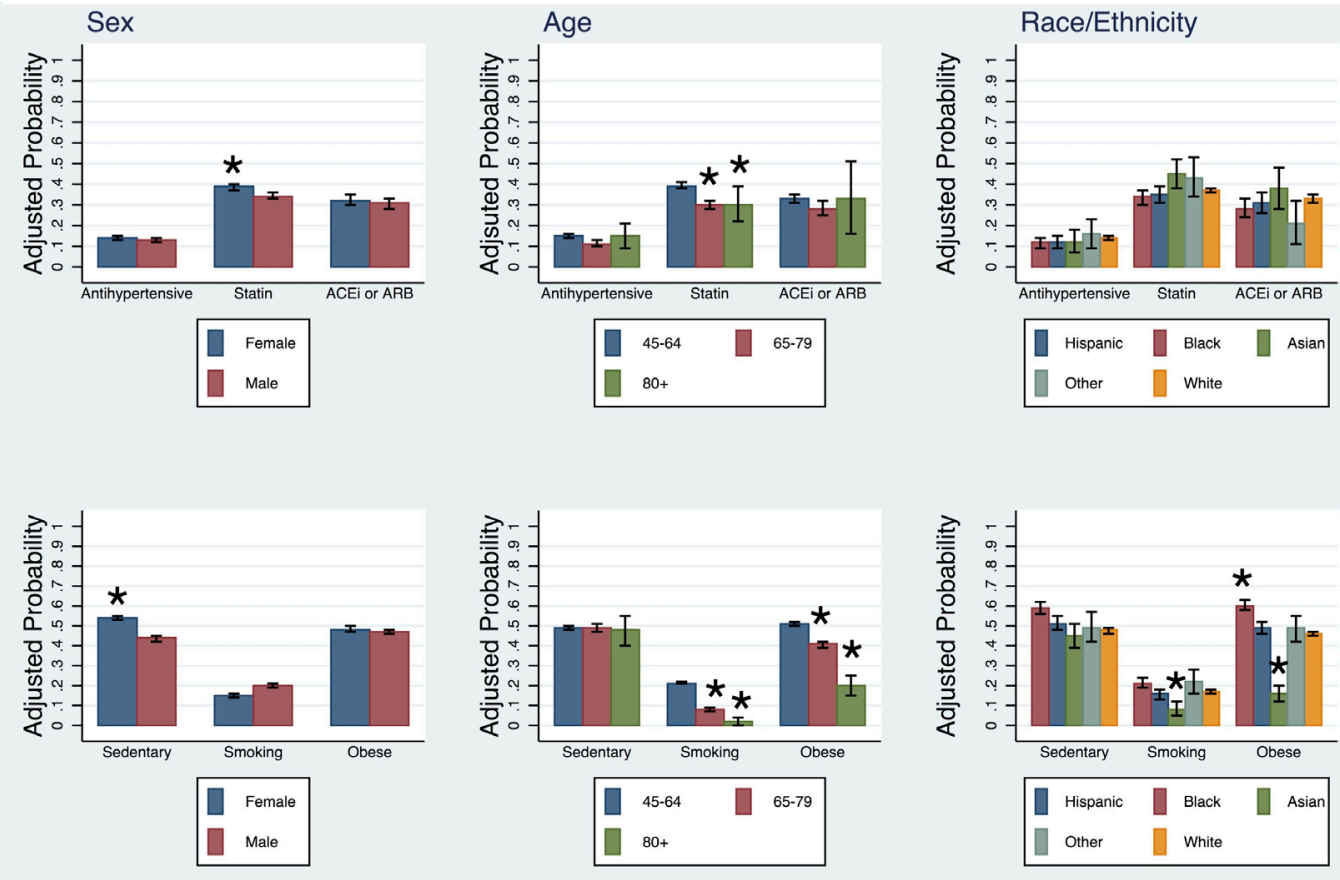

Figure 1 Preventive medical therapy and lifestyle practices at presentation, by sex, age and race/ethnicity. The bars represent covariate-adjusted probabilities of a preventive care gap, based on the multivariate models reported in table 2 . The reference groups for tests of statistical significance are male sex, 45-64 years old and White race/ethnicity. ACEi/ARB, angiotensinconverting enzyme inhibitor or angiotensin receptor blocker. We assessed antihypertensive use in hypertensives, statin use in dyslipidemics, and ACEi/ARB in diabetics. ${ }^{*} \mathrm{P}<0.05$

receive statins for dyslipidaemia (OR 1.31, 95\% CI 1.13 to 1.52). Compared with patients in the South, patients in all other US regions were less likely to be sedentary (Midwest: OR $0.58,95 \%$ CI 0.52 to 0.65 ; Northeast: OR $0.63,95 \%$ CI 0.55 to 0.72 ; West: OR $0.68,95 \%$ CI 0.61 to $0.77)$, and patients in the West were less likely to smoke (OR $0.76,95 \% \mathrm{CI} 0.65$ to 0.90 ), while patients in the Midwest were more likely to be obese (OR 1.23, 95\% CI 1.08 to 1.41$)$.

\section{DISCUSSION}

In the PROMISE trial population, we found that symptomatic patients presenting to their primary care physicians, cardiologists or other specialists with suspected CAD have a high prevalence of risk factors for adverse cardiovascular events, with many of these risk factors representing missed opportunities to improve preventive medical care and lifestyle practices. We identified populations that should be targeted for interventions based on their sex, age, race/ethnicity, socioeconomic status and geography. While some of the preventive care gaps were smaller in symptomatic patients at higher risk, others were larger or unassociated with baseline risk. Finally, our results support the notion that wider adherence to preventive medication and lifestyle practices may alter the epidemiology of chest pain presentations and reduce the incidence of evaluations for CAD. ${ }^{31}$

Our findings of gaps in preventive care and differences in these gaps across important sociodemographic characteristics complement the work of others. For example, in a study of patients with cardiovascular disease in the US Veterans Affairs health system, women were less likely to receive a statin than men. ${ }^{32}$ In another study of patients with peripheral artery disease, patients living in low socioeconomic status areas, as defined by median household income, were less likely to receive statins than patients living in higher socioeconomic status areas. ${ }^{28}$ Racial/ ethnic differences in exercise participation, smoking and obesity have also been reported. ${ }^{25}{ }^{33}$ However, our work extends and broadens the findings of these studies because (1) our study focused on actively symptomatic patients, whose presentation may be attributable to gaps in prevention and (2) we simultaneously accounted for a wider range of sociodemographic characteristics.

By assessing the relationship between baseline risk and preventive gaps, we showed that there was a trend towards lower preventive care gaps among symptomatic patients with high ASCVD scores, but higher preventive care gaps among symptomatic patients with elevated blood pressure. Our data also reflect more recent care preventive patterns across a broad geographic and socioeconomic 


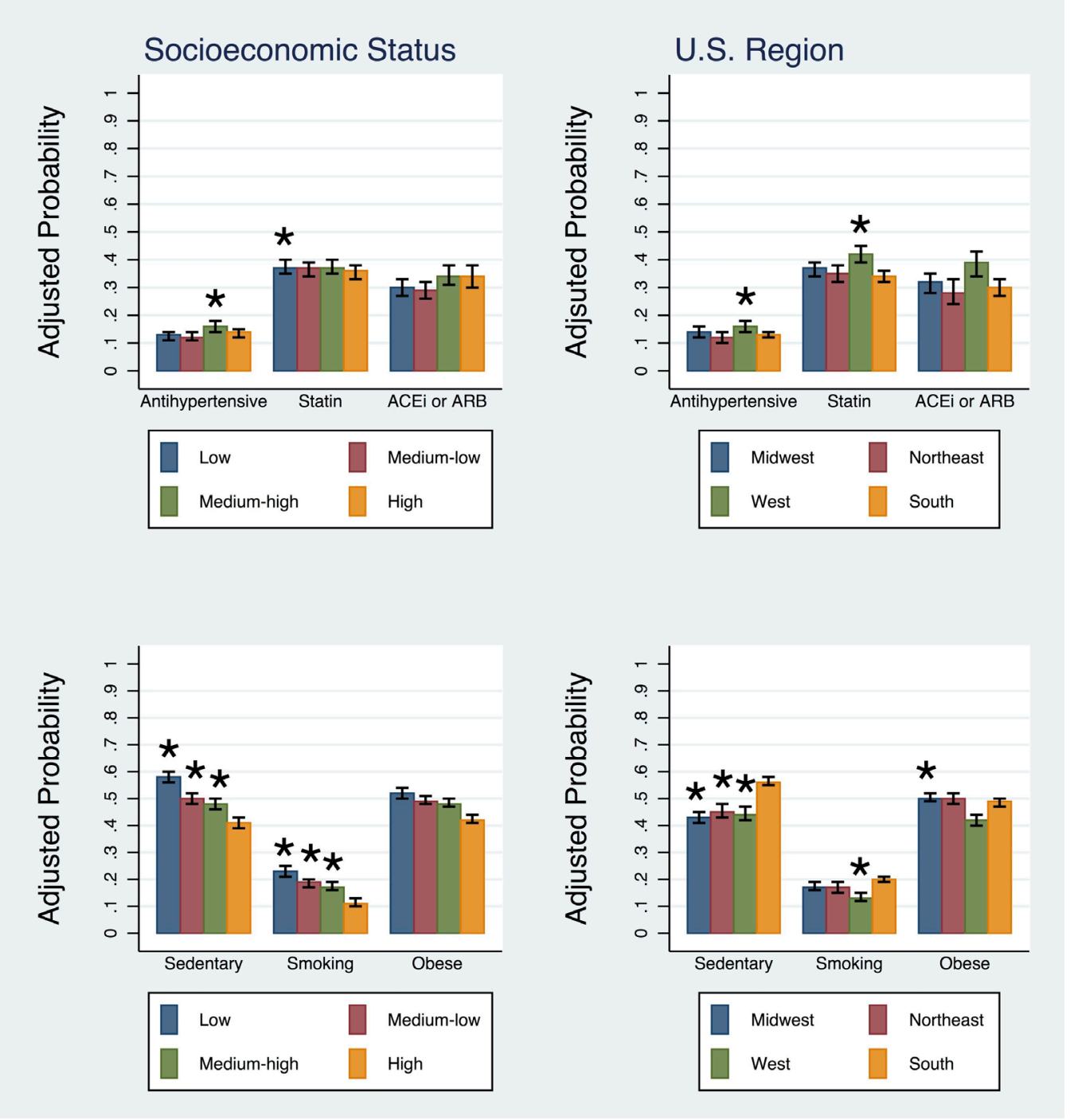

Figure 2 Preventive medical therapy and lifestyle practices at presentation. The bars represent covariate-adjusted probabilities of a preventive care gap, based on the multivariate models reported in table 2 . The reference groups for tests of statistical significance are high socioeconomic status and South region. ACEi/ARB, angiotensin-converting enzyme inhibitor or angiotensin receptor blocker. We assessed antihypertensive use in hypertensives, statin use in dyslipidemics, and ACEi/ARB in diabetics. ${ }^{*} \mathrm{P}<0.05$

sample. Our explicit inclusion of multiple racial/ethnic groups-particularly Asians-is also an advance for research in cardiovascular disease disparities, where previous comparisons have often been limited to Whites and Blacks only. ${ }^{29}{ }^{33-35}$ Our findings of disparities in preventive care are therefore more comprehensive and robust.

Similar to other studies of gaps in preventive care, our results highlight the importance of public health and policy initiatives aimed at bolstering primary prevention. Policy initiatives, such as the Million Hearts campaign, now leverage public-private partnerships and large investments in state and community programme to improve aspirin use in patients with $\mathrm{CAD}$, blood pressure control among patients with hypertension, cholesterol management and smoking cessation. ${ }^{36}{ }^{37}$ In addition, our findings reinforce the potential benefits of public and private policies that eliminate marginal cost-sharing for cholesterol and hypertension screening, obesity screening and counselling and smoking cessation services. ${ }^{38}$ Gaps in preventive care also highlight opportunities for making diagnostic testing a 'teachable moment' for symptomatic patients in this population-and for the primary care physicians and cardiologists caring for them. We did not examine the association of gaps in care with subsequent imaging, CAD diagnosis or invasive coronary angiography. 
In prior work, we showed that new initiation of an aspirin, statin, beta-blocker or ACEi/ARB was not associated with the rate of adverse cardiovascular events over a median follow-up period of 25 months in adjusted models. ${ }^{39} \mathrm{We}$ have also reported that absence of hypertension, dyslipidaemia and tobacco use are associated with a lower rate of adverse cardiovascular events. ${ }^{40}$ We also found that treatment gaps among patients with hypertension or diabetes were not associated with an increased risk of adverse cardiovascular events. In contrast, treatment gaps among patients with dyslipidaemia were associated with an increased risk of adverse cardiovascular events.

Our study has important limitations. There may have been patients whose hypertension or dyslipidaemia were well controlled with dietary changes and exercise alone. Among patients with diabetes, we did not have clinical information about albuminuria, so there may have been patients for whom the benefit of ACEi/ARB therapy was uncertain. In addition, our measures of preventive lifestyle practices were gathered through self-report; errors or inaccuracies in self-report could therefore affect our results. Our use of BMI as a surrogate for body fatness and obesity identification is also vulnerable to misclassification, since sex, age, race/ethnicity and muscle mass influence the relationship between BMI and excess fat. In terms of methodological strengths, our analyses included adjustments for multiple clinical characteristics and collected detailed race/ethnicity information. In addition, our study population was diverse in age, sex, income and geography. In terms of methodological weaknesses, because the study population was primarily composed of white patients, the study results may not be generalisable to all populations.

In conclusion, among contemporary, symptomatic patients presenting to primary care physicians, cardiologists and other specialists with suspected CAD, opportunities exist to bridge significant gaps in preventive care and lifestyle practices and reduce the incidence of future CAD. Differences by sex, age, race/ethnicity, socioeconomic status and geography tend to be modest but contribute to disparities and identify populations that should be targeted for interventions.

\section{Author affiliations \\ ${ }^{1}$ Division of General Internal Medicine and Health Services Research, David Geffen School of Medicine at UCLA, Los Angeles, California, USA \\ ${ }^{2}$ Department of Medicine, Duke Clinical Research Institute, Duke University School of Medicine, Durham, North Carolina, USA \\ ${ }^{3}$ National Heart, Lung, and Blood Institute, Bethesda, Maryland, USA \\ ${ }^{4}$ Louis Stokes Cleveland VA Medical Center, Cleveland, Ohio, USA \\ ${ }^{5}$ Oregon Health and Science University, Portland, Oregon, USA \\ ${ }^{6}$ Department of Radiology, Massachusetts General Hospital, Harvard Medical School, Boston, Massachusetts, USA}

Acknowledgements We are grateful for the contributions of the study participants and study team.

Contributors AC had full access to all the data in the study and takes responsibility for the integrity of the data and accuracy of the data analysis. Study concept and design: JL, PD. Acquisition, analysis or interpretation of data: all authors. Drafting of the manuscript: all authors. Critical revision of the manuscript for important intellectual content: all authors. Statistical analysis: AC, KL.
Funding The PROMISE trial was funded by National Heart, Lung, and Blood Institute grants R01 HL098237, R01 HL098236, R01 HL098305 and R01 HL098235. Dr Ladapo's work is supported by the National Heart, Lung,and Blood Institute (K23 HL116787) and he serves as a consultant to CardioDx, Inc. Dr Douglas has received grant support from HeartFlow and serves on a data and safety monitoring board for General Electric Healthcare; Dr Hoffmann has received grant support from Siemens Healthcare and HeartFlow; Dr Mark has received personal fees from Medtronic, CardioDx and St Jude Medical and grant support from Eli Lilly, Bristol-Myers Squibb, Gilead Sciences, AGA Medical, Merck, Oxygen Biotherapeutics and AstraZeneca. No financial disclosures were reported by the other authors of this paper.

Disclaimer The funding source had no role in the design and conduct of the study; collection, management, analysis and interpretation of the data; preparation, review or approval of the manuscript and decision to submit the manuscript for publication. The views expressed in this article do not necessarily represent the official views of the National Heart, Lung, and Blood Institute.

Competing interests None declared.

Ethics approval Duke University IRB.

Provenance and peer review Not commissioned; externally peer reviewed.

Data sharing statement All data are the property of the PROMISE Trial of Duke University. Study data availability is limited and available upon request.

Open Access This is an Open Access article distributed in accordance with the Creative Commons Attribution Non Commercial (CC BY-NC 4.0) license, which permits others to distribute, remix, adapt, build upon this work non-commercially, and license their derivative works on different terms, provided the original work is properly cited and the use is non-commercial. See: http://creativecommons.org/ licenses/by-nc/4.0/

(c) Article author(s) (or their employer(s) unless otherwise stated in the text of the article) 2017. All rights reserved. No commercial use is permitted unless otherwise expressly granted.

\section{REFERENCES}

1. Yusuf S, Hawken S, Ounpuu S, et al. Effect of potentially modifiable risk factors associated with myocardial infarction in 52 countries (the INTERHEART study): case-control study. Lancet 2004;364:937-52.

2. Stampfer MJ, Hu FB, Manson JE, et al. Primary prevention of coronary heart disease in women through diet and lifestyle. $N$ Engl J Med 2000;343:16-22.

3. Ladapo JA, Blecker S, Douglas PS. Physician decision making and trends in the use of cardiac stress testing in the United States: an analysis of repeated cross-sectional data. Ann Intern Med 2014;161:482-90.

4. Douglas PS, Hoffmann U, Patel MR, et al. Outcomes of anatomical versus functional testing for coronary artery disease. N Engl J Med 2015;372:1291-300.

5. Ladapo JA, Goldfeld KS, Douglas PS. Projected morbidity and mortality from missed diagnoses of coronary artery disease in the United States. Int J Cardiol 2015;195:250-2.

6. Young LH, Wackers FJ, Chyun DA, et al. Cardiac outcomes after screening for asymptomatic coronary artery disease in patients with type 2 diabetes: the DIAD study: a randomized controlled trial. JAMA 2009;301:1547-55.

7. Hachamovitch R, Nutter B, Hlatky MA, et al. Patient management after noninvasive cardiac imaging results from SPARC (Study of myocardial perfusion and coronary anatomy imaging roles in coronary artery disease). J Am Coll Cardiol 2012;59:462-74.

8. Cheezum MK, Hulten EA, Smith RM, et al. Changes in preventive medical therapies and CV risk factors after CT angiography. JACC Cardiovasc Imaging 2013;6:574-81.

9. Hulten E, Bittencourt MS, Singh A, et al. Coronary artery disease detected by coronary computed tomographic angiography is associated with intensification of preventive medical therapy and lower low-density lipoprotein cholesterol. Circ Cardiovasc Imaging 2014;7:629-38.

10. Douglas PS, Hoffmann U, Lee KL, et al. PROspective Multicenter Imaging Study for Evaluation of Chest Pain: rationale and design of the PROMISE trial. Am Heart J 2014;167:796-803.

11. Jackson R, Lawes CM, Bennett DA, et al. Treatment with drugs to lower blood pressure and blood cholesterol based on an individual's absolute cardiovascular risk. Lancet 2005;365:434-41.

12. Turnbull F, Neal B, Ninomiya T, et al. Effects of different regimens to lower blood pressure on major cardiovascular events in older 
and younger adults: meta-analysis of randomised trials. BMJ 2008;336:1121-3.

13. Weber MA, Schiffrin EL, White WB, et al. Clinical practice guidelines for the management of hypertension in the community a statement by the American Society of Hypertension and the International Society of Hypertension. J Hypertens 2014;32:3-15.

14. Rosendorff C, Black HR, Cannon CP, et al. Treatment of hypertension in the prevention and management of ischemic heart disease: a scientific statement from the American Heart Association Council for High Blood Pressure Research and the Councils on Clinical Cardiology and Epidemiology and Prevention. Circulation 2007;115:2761-88.

15. LaRosa JC, He J, Vupputuri S. Effect of statins on risk of coronary disease: a meta-analysis of randomized controlled trials. JAMA 1999;282:2340-6.

16. National Cholesterol Education Program Expert Panel on Detection. Evaluation, and Treatment of High Blood Cholesterol in Adults (Adult Treatment Panel III). Third Report of the National Cholesterol Education Program (NCEP) Expert Panel on Detection, Evaluation, and Treatment of High Blood Cholesterol in Adults (Adult Treatment Panel III) final report. Circulation 2002;106:3143-421.

17. Goff DC, Lloyd-Jones DM, Bennett G, et al. 2013 ACC/AHA guideline on the assessment of cardiovascular risk: a report of the American College of Cardiology/American Heart Association Task Force on Practice Guidelines. Circulation 20142014;129(25 Suppl 2):S49-73.

18. Pandya A, Sy S, Cho S, et al. Cost-effectiveness of 10-year risk thresholds for initiation of statin therapy for primary prevention of cardiovascular disease. JAMA 2015;314:142-50.

19. Patel A, MacMahon S, Chalmers J, et al. Effects of a fixed combination of perindopril and indapamide on macrovascular and microvascular outcomes in patients with type 2 diabetes mellitus (the ADVANCE trial): a randomised controlled trial. Lancet 2007;370:829-40.

20. Ruggenenti $P$, Fassi A, llieva AP, et al. Preventing microalbuminuria in type 2 diabetes. N Engl J Med 2004;351:1941-51.

21. Schrier RW, Estacio RO, Esler A, et al. Effects of aggressive blood pressure control in normotensive type 2 diabetic patients on albuminuria, retinopathy and strokes. Kidney Int 2002;61:1086-97.

22. Newman DJ, Mattock MB, Dawnay AB, et al. Systematic review on urine albumin testing for early detection of diabetic complications. Health Technol Assess 2005;9:163.

23. Adler Al, Stevens RJ, Manley SE, et al. Development and progression of nephropathy in type 2 diabetes: the United Kingdom Prospective Diabetes Study (UKPDS 64). Kidney Int 2003;63:225-32.

24. American Diabetes Association. (8) Cardiovascular disease and risk management. Diabetes Care 2015;38:S49-57.

25. Barbeau EM, Krieger N, Soobader MJ. Working class matters: socioeconomic disadvantage, race/ethnicity, gender, and smoking in NHIS 2000. Am J Public Health 2004;94:269-78.
26. Rathore SS, Berger AK, Weinfurt KP, et al. Race, sex, poverty, and the medical treatment of acute myocardial infarction in the elderly. Circulation 2000;102:642-8.

27. Rao SV, Schulman KA, Curtis LH, et al. Socioeconomic status and outcome following acute myocardial infarction in elderly patients. Arch Intern Med 2004;164:1128-33.

28. Subherwal S, Patel MR, Tang F, et al. Socioeconomic disparities in the use of cardioprotective medications among patients with peripheral artery disease: an analysis of the American College of Cardiology's NCDR PINNACLE Registry. J Am Coll Cardiol 2013;62:51-7.

29. Bucholz EM, Ma S, Normand SL, et al. Race, socioeconomic status, and life expectancy after acute myocardial infarction. Circulation 2015;132:1338-46.

30. Graubard BI, Korn EL. Predictive margins with survey data. Biometrics 1999;55:652-9.

31. Zoghbi GJ, Dorfman TA, Iskandrian AE. The effects of medications on myocardial perfusion. J Am Coll Cardiol 2008;52:401-16.

32. Virani SS, Woodard LD, Ramsey DJ, et al. Gender disparities in evidence-based statin therapy in patients with cardiovascular disease. Am J Cardiol 2015;115:21-6.

33. Folsom AR, Yatsuya $H$, Nettleton JA, et al. Community prevalence of ideal cardiovascular health, by the American Heart Association definition, and relationship with cardiovascular disease incidence. $J$ Am Coll Cardiol 2011;57:1690-6.

34. Hertz RP, Unger AN, Cornell JA, et al. Racial disparities in hypertension prevalence, awareness, and management. Arch Intern Med 2005;165:2098-104.

35. Diez Roux AV, Merkin SS, Arnett D, et al. Neighborhood of residence and incidence of coronary heart disease. N Engl J Med 2001;345:99-106.

36. Frieden TR, Berwick DM. The "Million Hearts" initiative-preventing heart attacks and strokes. N Engl J Med 2011;365:e27.

37. Sanghavi DM, Conway PH. Paying for prevention: a novel test of medicare value-based payment for cardiovascular risk reduction. JAMA 2015;314:123-4.

38. Koh HK, Sebelius KG. Promoting prevention through the Affordable Care Act. N Engl J Med 2010;363:1296-9.

39. Ladapo JA, Hoffmann U, Lee KL, et al. Changes in medical therapy and lifestyle after anatomical or functional testing for coronary artery disease. J Am Heart Assoc 2016;5:e003807.

40. Fordyce CB, Douglas PS, Roberts RS, et al. Prospective Multicenter Imaging Study for Evaluation of Chest Pain I. Identification of patients with stable chest pain deriving minimal value from noninvasive testing: The PROMISE minimal-risk tool, a secondary analysis of a randomized clinical trial. JAMA Cardiol 2017;2:400-8. 